\title{
Integrated Voltage Control and Line Congestion Management in Active Distribution Networks by Means of Smart Transformers
}

\author{
Giovanni De Carne, Marco Liserre \\ Chair of Power Electronics \\ Christian-Albrechts-University of Kiel \\ D-24143 Kiel, Germany \\ gdc@tf.uni-kiel.de,ml@tf.uni-kiel.de
}

\author{
Konstantina Christakou, Mario Paolone \\ Distributed Electrical Systems Laboratory \\ EPFL \\ Lausanne, Switzerland \\ konstantina.christakou@epfl.ch,mario.paolone@epfl.ch
}

\begin{abstract}
Within the context of Active Distribution Networks (ADNs), smart transformers represent very powerful devices able to provide fast and efficient control actions with respect to different ADNs ancillary services. This paper discusses the benefits, in terms of ADNs voltage and line flows controls, achieved by interfacing distributed generators with the power grid by means of a smart transformer. Among several benefits, these devices allow for a phase-per-phase control of the generators active and reactive power injections. This peculiarity enables to deploy new control schemes that are analyzed and discussed in the paper with reference to a case study based on a modified IEEE 34 node test distribution feeder.
\end{abstract}

Index Terms-Smart transformer, Solid State Transformer, Smart Grids, Distributed Generation, Voltage Control, Current Congestion Management.

\section{INTRODUCTION}

The last-decade penetration of Distributed Energy Resources (DERs) has called for new technologies to be available in electrical distribution systems. These systems, designed as passive networks aimed at carrying energy from centralized power plants towards customers, are evolving to active networks, power-decentralized, and able to exchange not only power in every direction, but also information and data.

Within the context of Active Distribution Networks (ADNs), many known distributed generation (DG) technologies, like wind, photovoltaic and combined heat-and-power units, have largely improved their performances in terms of capacity and conversion efficiency. The introduction of these new power generation technologies has forced Distribution Network Operators (DNOs) to increase the monitoring level of their networks by using different metering infrastructures coupled with advanced Supervisory Control and Data Acquisition systems (SCADA) [1]. The next step is represented by the deployment of pervasive control schemes able to couple optimal control functions with the distributed DERs.

In this respect, DNOs aim at upgrading their ADNs in order to gain more control of their systems. In this direction, secondary substations and transformers are vital elements. Voltage control in presence of massive integration of volatile renewables, management of line congestions, and power quality improvement, are only few of the many tasks that the DERs might be requested to provide [2-5].

The smart transformer (ST) is expected to have the same performances of the traditional transformer and provide, at the same time, network ancillary services and exchange of information (i.e., data aggregator). By using a suitably defined centralized optimal control algorithm, this work aims at demonstrating that smart transformers, interfacing small generation units, allow us to have better performance in terms of voltage control and current congestion management than a traditional transformer. The main feature of a smart transformer is to control independently the power injection of each phase, representing a great advantage in an unbalanced ADN.

The rest of the paper is organized as follows: in section II, a brief introduction is given regarding the DERs' impact and the solution offered by using a ST. The same section describes the state of the art regarding voltage and current control in low voltage networks. Section III is dedicated to the mathematical formulation of a suitably defined optimal control algorithm based on the work presented in [6]. In section IV the algorithm is evaluated through application examples involving a modified IEEE 34 node test feeder. Finally, section V is dedicated to the conclusions.

\section{RELATED WORK}

\section{A. Voltage and Line Congestion Controls in ADNs}

Traditionally, voltage control is performed by means of OnLoad Tap Changers (OLTCs) [7] located in the transformers of primary substations or by controlling the reactive power injections of devices such as Static VAr Compensators (SVCs) [8].

In the first case, OLTCs have a discrete controller that allows the voltage ratio set-point to be changed, thus modifying the voltage magnitude. OLTCs, in general, allow for following properly the load trend in the network, mostly in stressed conditions and under the presence of DG (e.g., [7], [911]). However, the OLTC-based voltage control represents a centralized method and does not allow for a flexible control 
of the voltage in the different network buses. Furthermore, the number of OLTC switches allowed is restricted and the voltage range that can be controlled is limited [12]. Thus, these devices are not good candidates for an optimal and fast voltage control of ADNs, where one of the main characteristics is the penetration of highly volatile renewable energy resources such as PVs.

As far as the Static VAr Compensator (SVC) and capacitors banks are concerned, they provide a continuous (SVC) or discrete control (capacitors banks) for the injection of reactive power in the network [13-15]. This has a local effect on the voltage profile of the buses where these devices are connected. Furthermore, due to the non-negligible $\mathrm{R} / \mathrm{X}$ ratio of the network lines of distribution grids, voltage control requires active power management in addition to reactive power control [16].

Currently, in presence of line ampacity violations, no optimal control solution has been established. Generally, when the network is overloaded, the DNOs curtail the active power. In the active power curtailment the loads and/or DERs are curtailed in strategic points of the network until the current flows lie again within their allowed limits [17].

The aforementioned approaches represent an extreme solution when dealing with voltage and current flows violations as they are not cost effective and they do not provide any supplementary service to the network, such as data exchange.

\section{B. The Smart Transformer Concept}

All the aforementioned issues can be tackled by the so called smart transformer as it can control the power injections of DERs and manage the current congestion at the same time. The concept of the ST is shown in Fig. 1. It can provide several services to the grid, such as voltage control, increase of hosting capability in presence of distributed resources (PHEVs, BEVs, PVs, etc.) [18], reduction of current flows and losses in the distribution lines, and reduction of harmonic profiles. ST could work as an interface between voltage levels being a smart grid control center for the lower voltage network and at the same time a single control point for the higher voltage systems. The ST can control locally the low voltage grid, using the data of the local loads, and can aggregate and exchange data with the higher voltage network. This concept allows, in principle, to avoid data congestion in the network, and to reduce the communication burden, that is equivalent to a reduction of communication infrastructures.

Finally, the ST offers an optimal management of the harmonics, created by power electronics of DERs. ST avoids the harmonics injection, due to the physical separation of the circuit by means of a DC link

Several different architectures of STs have been proposed in [19]. In this work, we have chosen the topology depicted in Fig. 2, due to the presence of a common DC link in the LV side, and separate DC links in the HV side. Among the configurations in [19], the latter represents the best solution for a per-phase control.

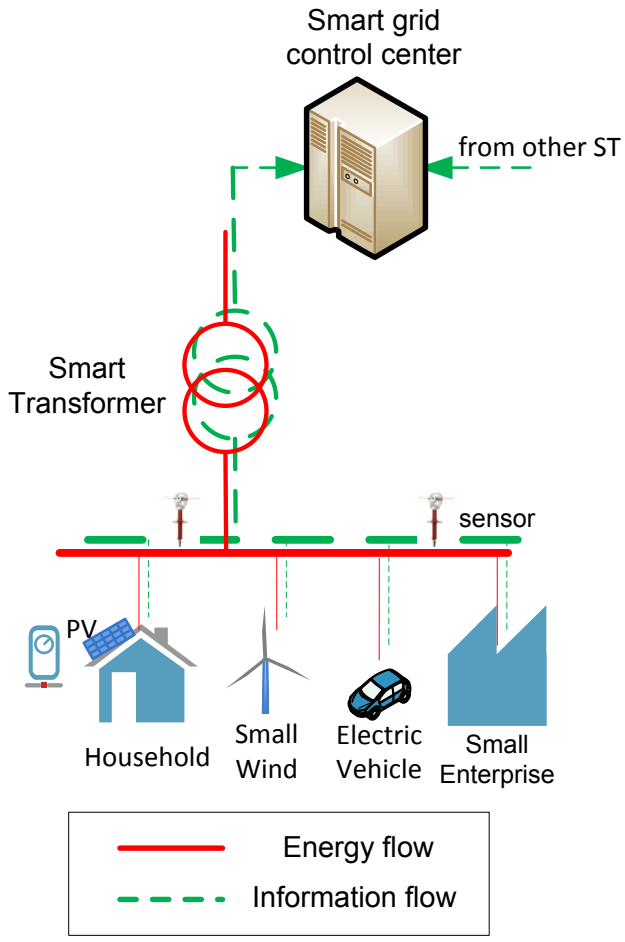

Fig. 1. Smart Transformer concept.

Indeed, this configuration allows us to distribute the generated power according to the network needs as it is possible to provide power to each phase independently. It is worth noting that the maximum power flow in each phase is limited by the size of the DC/DC converter, the DC link, and the single phase inverter. In this paper, the maximum power for each phase has been assumed to be one third of the maximum threephase power in order to satisfy the converter's capabilities. The generators are considered to be directly connected to the LV side and interfaced with the distribution network in the HV side by means of the ST.

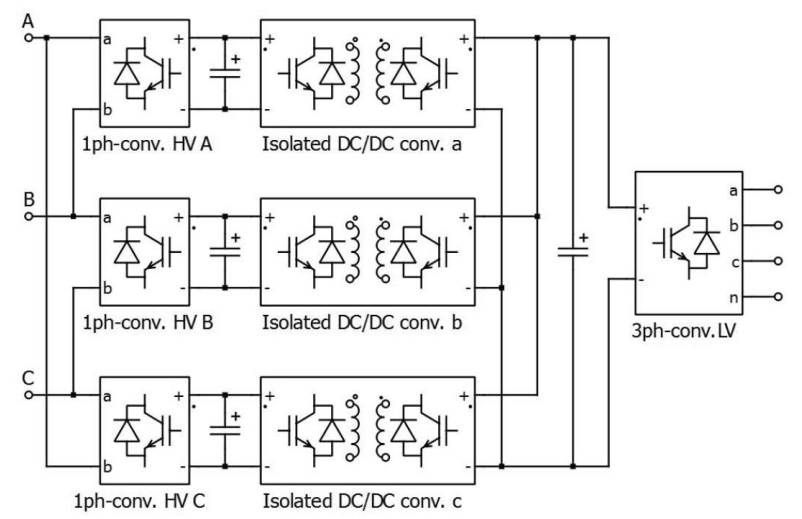

Fig. 2. Smart Transformer topology adapted from [19]. 


\section{MATHEMATICAL Formulation OF THE Optimal CONTROL PROBLEM}

\section{A. Proposed Algorithm}

The proposed algorithm aims at using a ST that allows injecting power at each phase independently of the other two phases, in order to perform voltage and lines congestion control. Normally, with a traditional transformer the DNOs are not allowed to modify the injection of active and reactive powers of a single phase even if the network is highly unbalanced. However, with a ST, the DNO can envision to adapt the per-phase power injection as a function of the network needs.

The flow chart shown in Fig. 3 summarizes the steps of the proposed algorithm. At each time step (in what follows we have assumed a control time step of 15 minutes) the DNO observes the state of the network, i.e. voltage magnitude and phase at each bus, by means of a state estimation process (e.g., [20], [21]). Then, the sensitivity coefficients of the voltage and current flows [6] with respect to the generators' active and reactive power injections are computed, as explained in the next section. Subsequently, an on-line centralized controller solves an optimization problem in order to obtain the new active and reactive power set points of the generators that guarantee a network voltage and current profile within acceptable limits for the safe operation of the grid. Finally, the computed $(P, Q)$ set points are sent to the DG units. This operation is repeated at every time step, until the end of the time window.

\section{B. On-line Centralized Controller's Actions}

A network is considered with $n_{b}$ buses, $n_{l}$ lines and $M$ generators. At every time step, the voltage and current sensitivity coefficients with respect to active and reactive power injections at each bus are defined as follows ${ }^{1}$ :

$$
\begin{array}{cc}
K_{P, i l}(t)=\frac{\partial\left|\bar{E}_{i}\right|}{\partial P_{l}}(t) & K_{Q, i l}(t)=\frac{\partial\left|\bar{E}_{i}\right|}{\partial Q_{l}}(t) \\
H_{P, k l}(t)=\frac{\partial \bar{I}_{k}}{\partial P_{l}}(t) & H_{Q, k l}(t)=\frac{\partial \bar{I}_{k}}{\partial Q_{l}}(t)
\end{array}
$$

These sensitivities are computed on-line by solving a linear system of equations as presented in [6].

The sensitivities allow us to obtain the following linear relations between the variation of the bus voltages and line currents and the active and reactive power injections at the network buses $\Delta P_{i}, \Delta Q_{i}$ :

$$
\begin{gathered}
\Delta\left|\bar{E}_{i}\right| \approx K_{P_{i}} \Delta P+K_{Q_{i}} \Delta Q \triangleq\left(K_{P, Q}(t) \Delta(P, Q)\right)_{i} \\
\Delta \bar{I}_{k} \approx H_{P_{k}} \Delta P+H_{Q_{k}} \Delta Q \triangleq\left(H_{P, Q}(t) \Delta(P, Q)\right)_{k}
\end{gathered}
$$

\footnotetext{
${ }^{1}$ In what follows complex quantities are denoted with a bar above (e.g., $\left.\bar{I}\right)$
}

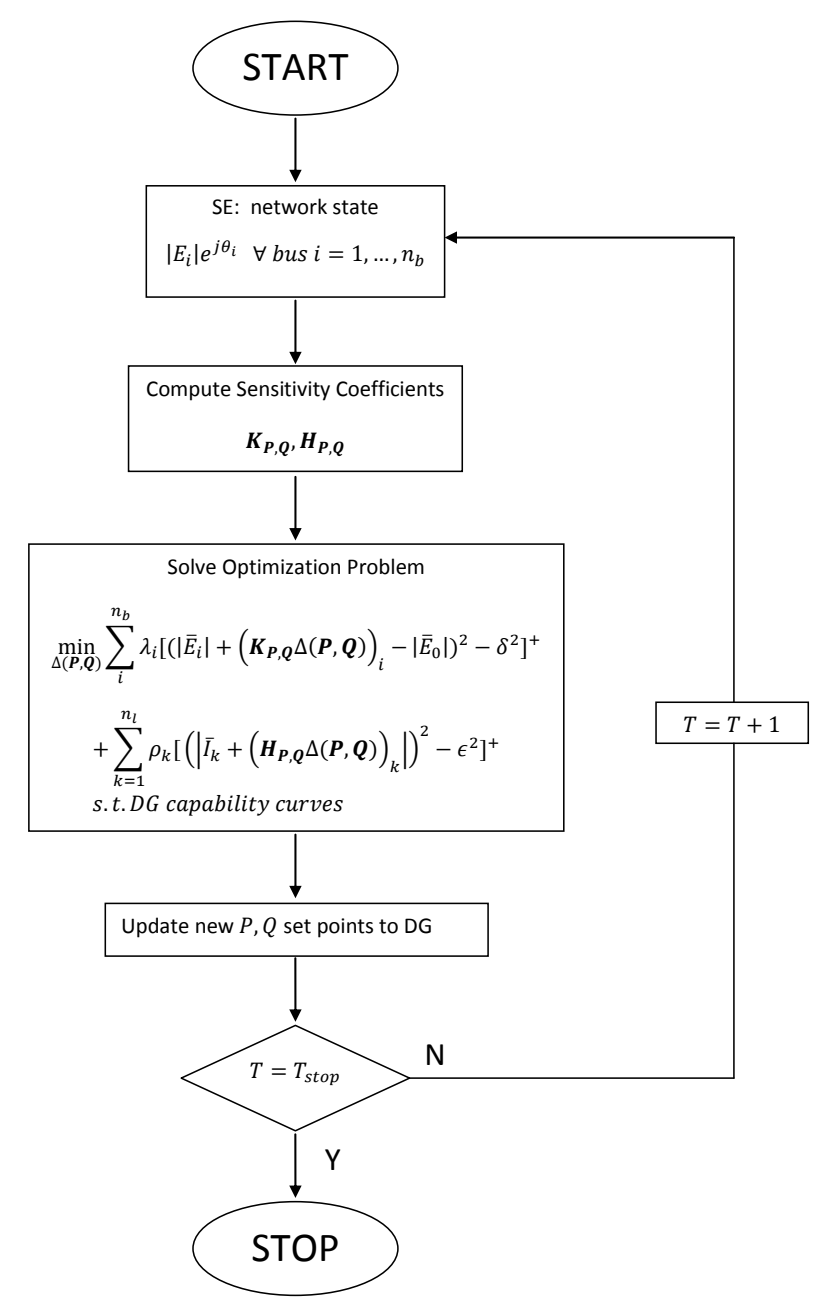

Fig. 3. Flow chart of the proposed algorithm.

In the above set of equations $K_{P_{i}}=\left[K_{P, i 1}, \ldots, K_{P, i M}\right]$, $K_{Q_{i}}=\left[K_{Q, i 1}, \ldots, K_{Q, i M}\right], H_{P_{k}}=\left[H_{P, k 1}, \ldots, H_{P, k M}\right]$, and $H_{Q_{k}}=\left[H_{Q, k 1}, \ldots, H_{Q, k M}\right]$.

The proposed approach allows us to perform both voltage control and lines congestion management by controlling the active and reactive power variations of the controllable resources in the network. In this direction, at each time step a minimization problem is solved. The corresponding objective function of the problem is as follows:

$$
\begin{array}{r}
\min _{\Delta(P, Q)} \sum_{i=1}^{n_{b}} \lambda_{i}\left[\left(\left|\bar{E}_{i}\right|+\left(K_{P, Q}(t) \Delta(P, Q)\right)_{i}-\left|\bar{E}_{o}\right|\right)^{2}-\delta^{2}\right]^{+} \\
+\sum_{k=1}^{n_{l}} \rho_{k}\left[\left(\left|\bar{I}_{k}+\left(H_{P, Q}(t) \Delta(P, Q)\right)_{k}\right|\right)^{2}-\epsilon^{2}\right]^{+}
\end{array}
$$

where we have used the notation $[a]^{+} \triangleq \max (a, 0)$ and $\lambda_{i}$ and $\rho_{k}$ are weighting the terms of the objective function that correspond to voltage and current control respectively. We have fixed $\lambda_{i}=\rho_{k}=0.5$, in order to account equally 


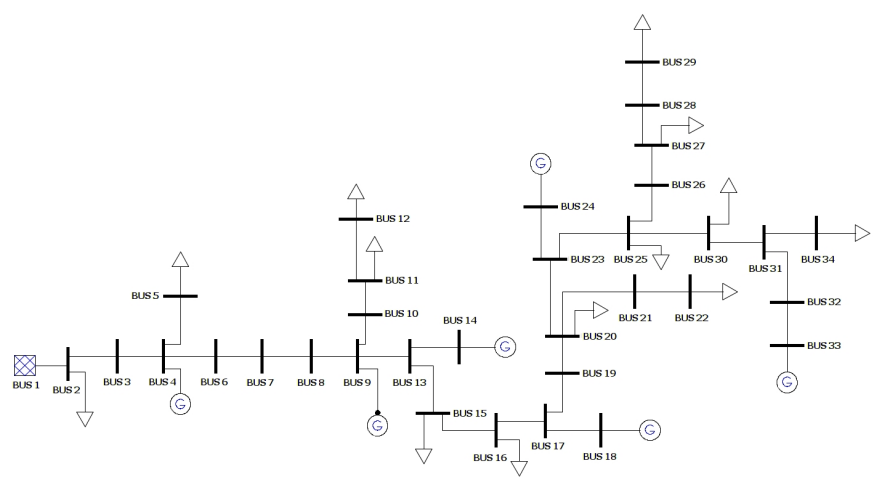

Fig. 4. Modified IEEE 34 node test feeder, adapted from [22].

for the effect of voltage and current in the optimization problem. The constants $\delta$ and $\epsilon$ represent the voltage and current thresholds respectively, which define the bounds above which the controller optimizes the voltage and the current flows. In this case $\delta=0.03$ p.u. and $\epsilon=0.8$ p.u. of line ampacities, i.e., only the buses with a voltage below 0.97 p.u. or above 1.03 p.u., and only the lines with a current above $80 \%$ of their ampacity value, will be taken into account in the optimization problem. This avoids the minimization of the voltage deviations and the current flows when they are within acceptable limits imposed by DNOs.

\section{Performance Evaluation of The Proposed ALGORITHM}

\section{A. Network Settings}

For the evaluation of the proposed algorithm we have considered a modified IEEE 34 node test feeder as shown in Fig. 4. The modifications are (i) exclusion of the regulators and shuntcapacitors to make the network weaker, (ii) placement of 6 generators, with a maximum three-phase power of $900 \mathrm{~kW}$, located in buses $4,9,14,18,24$, and 34 .

The ampacity of the network lines is fixed to 100A, which is a typical value for lines in distribution networks. The loads are unbalanced, and the total load profile of the network is depicted in Fig. 5 in terms of $24 \mathrm{hr}$ active and reactive power injections for each phase. As it can be observed from Fig. 5, the phases $c$ and $a$ are more overloaded than the phase $b$. Fig. 6 shows the generators' scheduling when no voltage and lines congestion control action is applied. In this case we have considered that the generators inject the same amount of power per-phase (balanced injection). Therefore, for the sake of brevity, Fig. 6 shows only the injected power of phase $a$.

\section{B. Generators Settings}

We have considered two different test cases for the settings of each of the six generators that are present in the network. In the first case (Case A) each generator is equipped with a traditional transformer. Their overall output is assumed to have a rectangular capability curve with 0.95 lag-to-lead range.
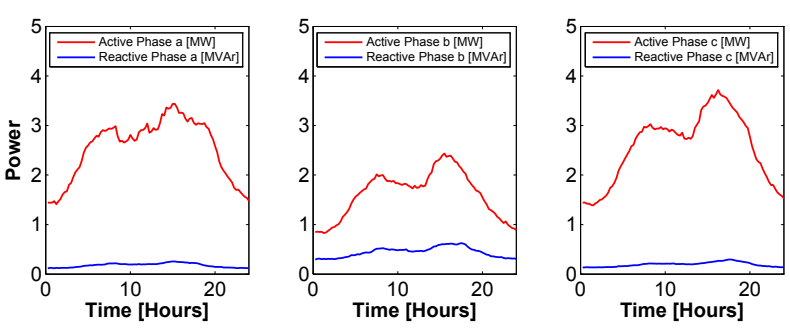

Fig. 5. Total daily load profile in the network.

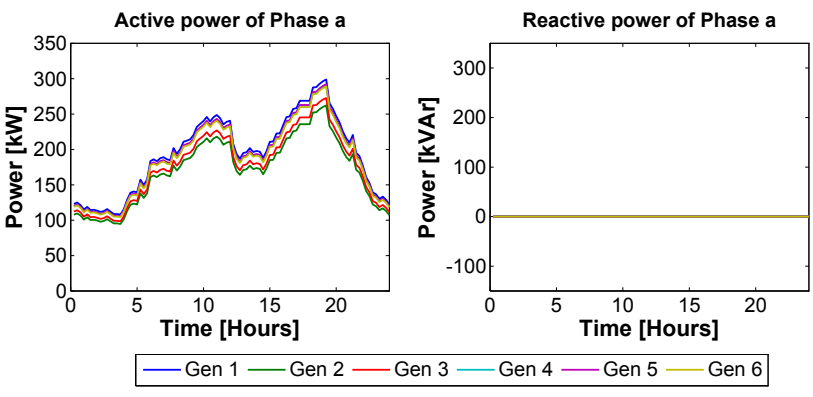

Fig. 6. Total daily generators scheduling.

For the second case (Case B) a smart transformer interfaces each generator with the network. Additionally, the smart transformer output is assumed to have a triangular capability curve as depicted in Fig. 7. The maximum range varies between 0.95 p.u. lag and 0.925p.u. lead, as suggested in [23] for photovoltaic and wind power plants in MV installations in Germany. In both test cases the transformers' size is 1.5MVA.

\section{Voltage and Lines Congestion Control}

In this section the DNO performs voltage and lines congestion management by scheduling the DG units available in the network. In order to better understand the benefits of using a ST versus a traditional one for these types of ancillary services, we show in Fig. 8 the per-phase network current and voltage

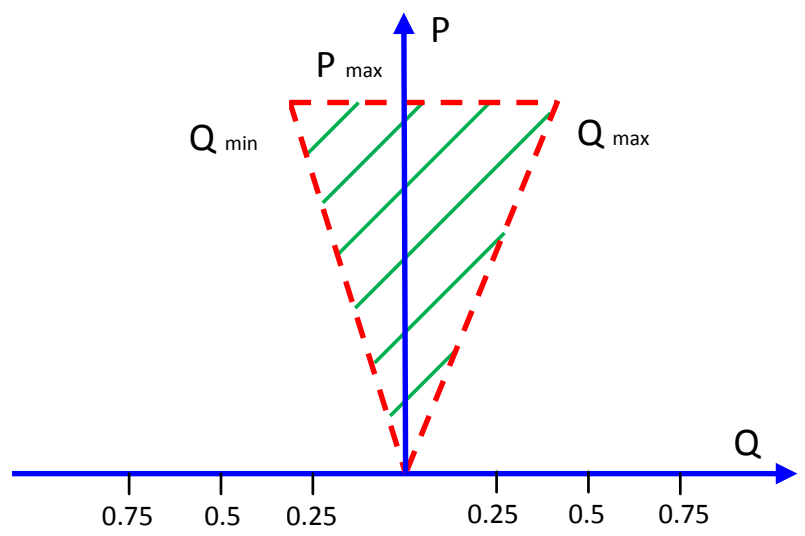

Fig. 7. Test Case B capability curve of the generators adopted from [23]. 


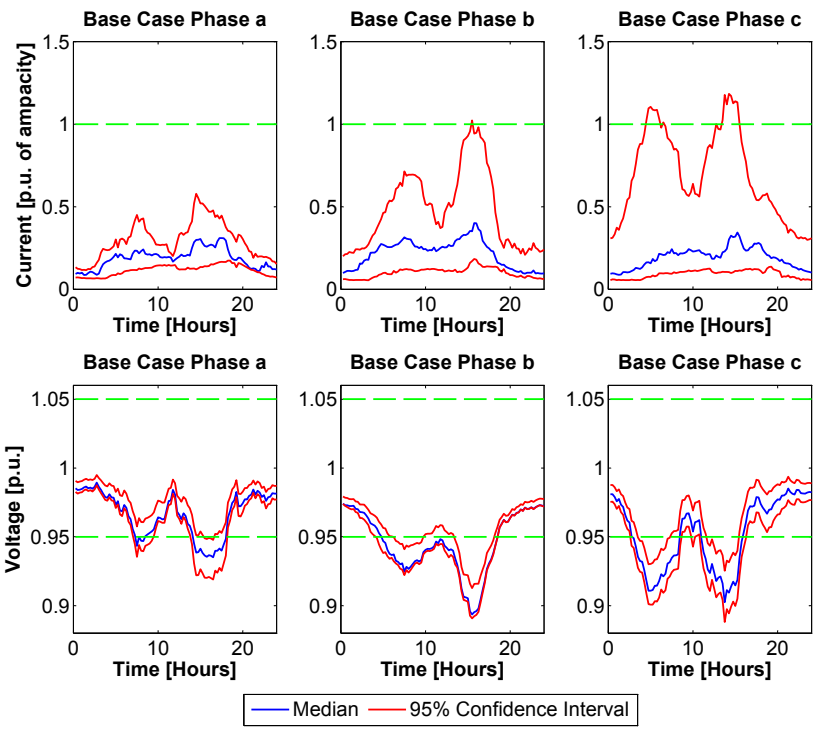

Fig. 8. Base case $24 \mathrm{hr}$ per-phase current and voltage profiles (median and $95 \%$ confidence intervals).

profiles, for the base case load and generators' profiles shown in Fig. 5 and Fig. 6.

For the sake of brevity we show in blue the median of the voltage and current values of the network nodes and lines respectively, and in red lines the corresponding $95 \%$ confidence intervals. The light green dashed lines represent the voltage as well as the current limits. For the voltage, these limits are set to \pm 0.05 p.u. of the network voltage rated value and for the current, to 1p.u. of the line ampacity. Due to the heavy loading of the network there are periods during the day when both the voltage and the current profiles exceed the allowed limits.

In order to avoid a possible curtailment of the load the DNO is forced to re-dispatch the generators. In Case A the DNO uses a traditional transformer interfaced with the generators, and thus the generators inject the same amount of power per phase. For this case, Fig. 9 shows the resulting network current and voltage profiles.

As it can be observed, the proposed algorithm improves the voltage and current profiles in the network, particularly in phase $a$, but the control action is not sufficient to manage efficiently the voltage control and current congestion management in phases $b$ and $c$, due to the balanced control of the generators' output. The corresponding scheduling of the generators' active and reactive power injections for the same test case is shown in Fig. 10.

Fig. 10 shows that the active and reactive powers are almost saturated for all six generators almost throughout the $24 \mathrm{hr}$ period. This is explained by the fact that even though not all phases are equally loaded, the algorithm is forced to adapt the set points of the generators to the most overloaded phases, which are phases $b$ and $c$.

In Case B, the DNO deploys a smart transformer to interface

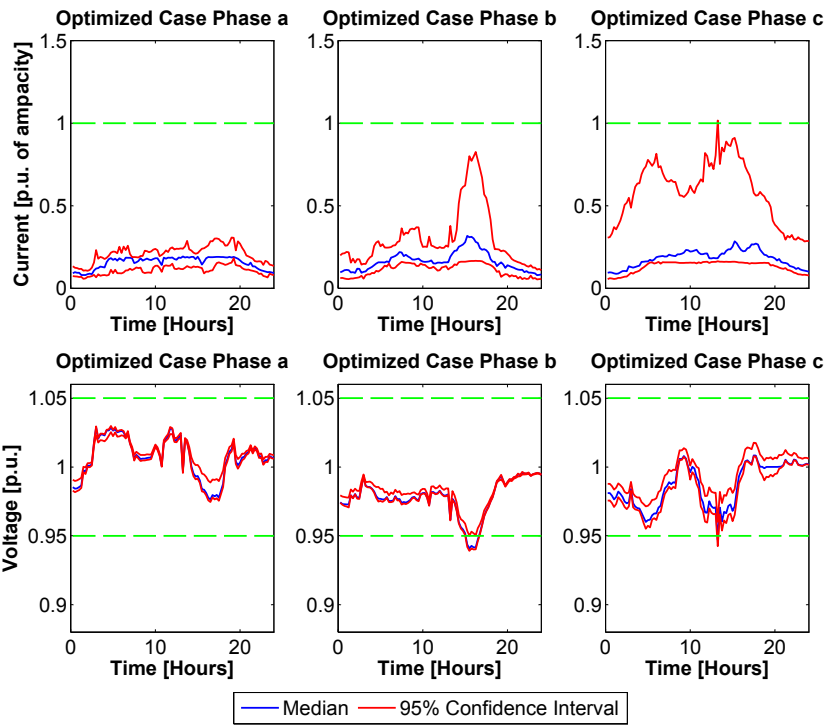

Fig. 9. Test Case A 24hr per-phase current and voltage profiles (median and $95 \%$ confidence intervals).

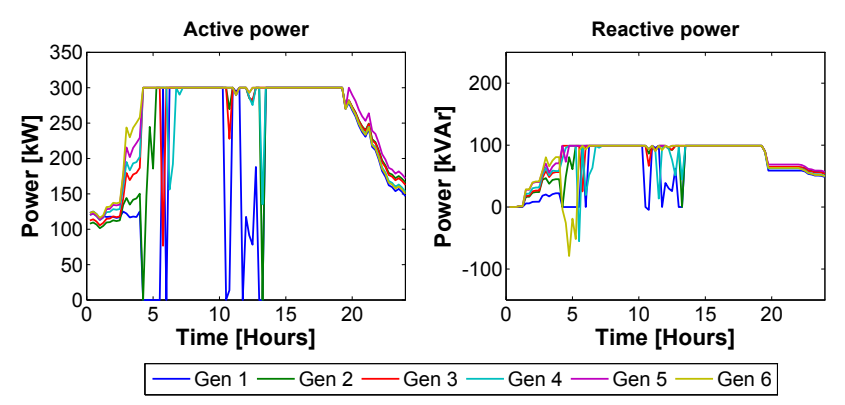

Fig. 10. Test Case A 24hr generators' active and reactive power scheduling.

the generators with the distribution network. The STs can perform a phase-per-phase control. In this way, the power injection of each phase is scheduled separately throughout the day, independently from the other two phases. Applying the proposed algorithm to this test case, results in the profiles of the network currents and voltages as shown in Fig. 11. The smart transformer is able to bring the voltage and current profiles of all 3 phases within the acceptable limits for safe operation.

For the same test case, Fig. 12 shows the $24 \mathrm{hr}$ scheduling of the active and reactive power injections for all six generators. The phase-per-phase control results in different profiles with respect to Case A. As it can be observed, only the active power profiles of phase $b$ and $c$ are saturated throughout the day. On the contrary, phase $a$ requires less power during the day with respect to the traditional transformer case.

Concerning the reactive power profiles, we obtain a significantly different distribution along the three phases. The three phases are less overloaded than in Case A, and the reactive power profiles are not saturated during the day. 


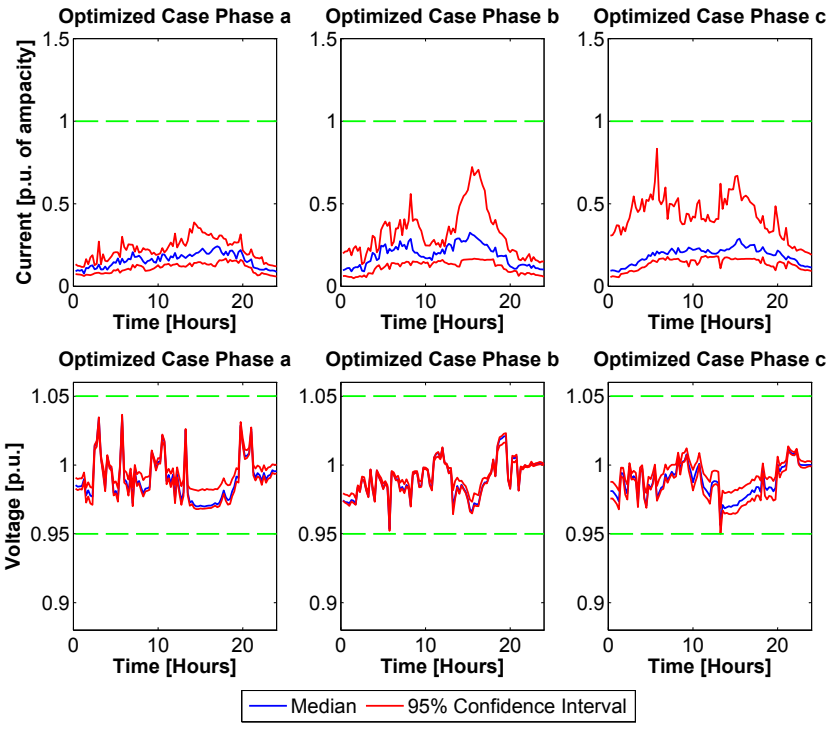

Fig. 11. Test Case B 24hr per-phase current and voltage profiles (median and $95 \%$ confidence intervals).
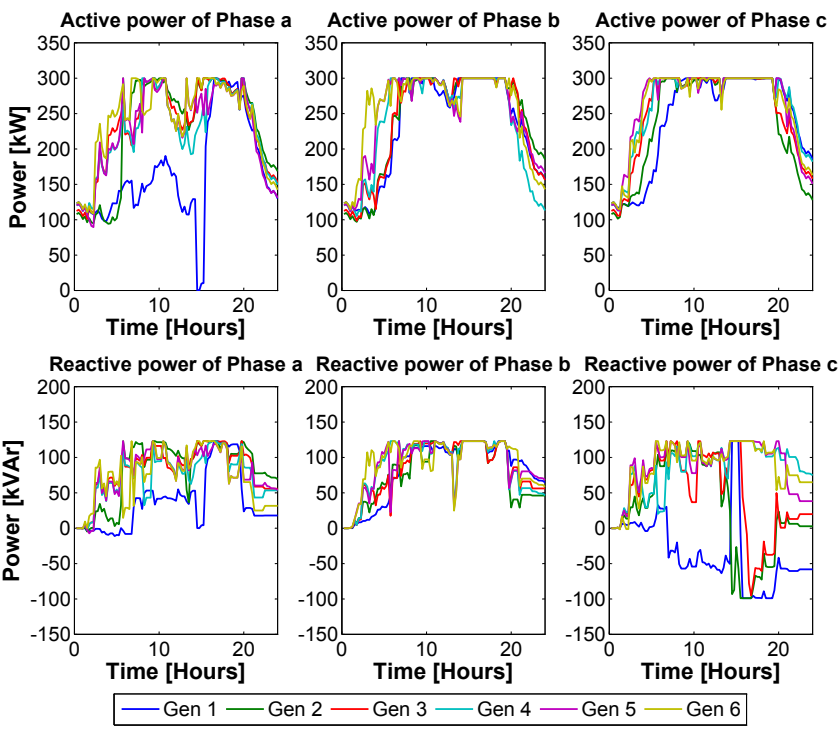

Fig. 12. Test Case B 24hr generators' active and reactive power scheduling.

\section{CONCLUSIONS}

By using a suitably defined centralized optimal control algorithm based on a previous work, this paper has revealed the advantages of interfacing DERs in ADNs using STs in order to provide improved grid ancillary services. ST has proved to be a flexible tool for performing voltage control and lines congestion management. The results presented in this work indicate that, the ST represents a promising way to manage low and medium voltage networks.

\section{ACKNOWLEDGEMENTS}

This study was funded under the ERC Consolidator Grant 2014-2019 in the project "Highly Efficient and Reliable smart Transformer" (HEART).

\section{REFERENCES}

[1] K. Katsaros, W. Chai, N. Wang, G. Pavlou, H. Bontius, and M. Paolone, "Information-centric network for machine-to-machine data delivery - a case study in smart grid applications," IEEE Network Magazine, Special Issue on Information-Centric Networking Beyond Baseline Scenarios: Research Advances and Implementation, (to appear).

[2] T. Vandoorn, J. D. M. De Kooning, B. Meersman, J. Guerrero, and L. Vandevelde, "Voltage-based control of a smart transformer in a microgrid," IEEE Trans. on Industrial Electronics, vol. 60, no. 4, pp. 1291-1305, April 2013.

[3] P. Kadurek, J. F. G. Cobben, and W. Kling, "Smart transformer for mitigation of voltage fluctuations in MV networks," in 10th International Conference on Environment and Electrical Engineering (EEEIC), May 2011, pp. 1-4.

[4] J. C. Kester, P. J. Heskes, J. Kaandorp, J. Cobben, G. Schoonenberg, D. Malyna, E. C. de Jong, B. J. Wargers, and A. Dalmeijer, "A smart MV/LV-station that improves power quality, reliability and substation load profile," in 20th International Conference and Exhibition on Electricity Distribution - Part 1, CIRED, June 2009, pp. 1-4.

[5] P. Kadurek, J. F. G. Cobben, and W. Kling, "Smart MV/LV transformer for future grids," in International Symposium on Power Electronics Electrical Drives Automation and Motion (SPEEDAM), June 2010, pp. 1700-1705.

[6] K. Christakou, J.-Y. LeBoudec, M. Paolone, and D.-C. Tomozei, "Efficient computation of sensitivity coefficients of node voltages and line currents in unbalanced radial electrical distribution networks," IEEE Trans. on Smart Grid, vol. 4, no. 2, pp. 741-750, June 2013.

[7] C. Gao and M. Redfern, "A review of voltage control techniques of networks with distributed generations using on-load tap changer transformers," in 45th International Universities Power Engineering Conference (UPEC), Aug 2010, pp. 1-6.

[8] J.-H. Chen, W.-J. Lee, and M.-S. Chen, "Using a static var compensator to balance a distribution system," IEEE Trans. on Industry Applications, vol. 35, no. 2, pp. 298-304, Mar 1999.

[9] K. Muttaqi, A. Le, M. Negnevitsky, and G. Ledwich, "A coordinated voltage control approach for coordination of OLTC, voltage regulator and DG to regulate voltage in a distribution feeder," in Industry Applications Society Annual Meeting, IEEE, Oct 2013, pp. 1-8.

[10] C. Reese, C. Buchhagen, and L. Hofmann, "Voltage range as control input for OLTC-equipped distribution transformers," in Transmission and Distribution Conference and Exposition (TD), IEEE PES, May 2012, pp. 1-6.

[11] X. Liu, A. Aichhorn, L. Liu, and H. Li, "Coordinated control of distributed energy storage system with tap changer transformers for voltage rise mitigation under high photovoltaic penetration," IEEE Trans. on Smart Grid, vol. 3, no. 2, pp. 897-906, June 2012.

[12] S. Virayavanich, A. Seiler, C. Hammer, and K.-H. Weck, "Reliability of on-load tap changers with special consideration of experience with delta connected transformer windings and tropical environmental conditions," in Cigré, 1996.

[13] P. Bogonez-Franco, J. Balcells, O. Junyent, and J. Jorda, "SVC model for voltage control of a microgrid," in IEEE International Symposium on Industrial Electronics (ISIE), June 2011, pp. 1645-1649.

[14] M. Abdel-Rahman, F. Youssef, and A. Saber, "New static var compensator control strategy and coordination with under-load tap changer," IEEE Trans. on Power Delivery, vol. 21, no. 3, pp. 1630-1635, July 2006.

[15] A. Vukojevic, P. Frey, M. Smith, and J. Picarelli, "Integrated volt/var control using single-phase capacitor bank switching," in Innovative Smart Grid Technologies (ISGT), IEEE PES, Feb 2013, pp. 1-7.

[16] K. Christakou, D.-C. Tomozei, M. Bahramipanah, J.-Y. Le Boudec, and M. Paolone, "Primary voltage control in active distribution networks via broadcast signals: The case of distributed storage," IEEE Trans. on Smart Grid, (to appear).

[17] P. Rao and K. Rao, "An efficient load shedding algorithm for radial systems," in Conference on Convergent Technologies for the Asia-Pacific Region, TENCON, vol. 2, Oct 2003, pp. 771-774. 
[18] S. Huang, J. Pillai, M. Liserre, and B. Bak-Jensen, "Improving photovoltaic and electric vehicle penetration in distribution grids with smart transformer," in 4th IEEE/PES Innovative Smart Grid Technologies Europe (ISGT EUROPE), Oct 2013, pp. 1-5.

[19] R. Pena-Alzola, G. Gohil, L. Mathe, M. Liserre, and F. Blaabjerg, "Review of modular power converters solutions for smart transformer in distribution system," in Energy Conversion Congress and Exposition (ECCE), IEEE, Sept 2013, pp. 380-387.

[20] I. Roytelman and S. M. Shahidehpour, "State estimation for electric power distribution systems in quasi real-time conditions," IEEE Trans. on Power Delivery, vol. 8, no. 4, pp. 2009-2015, Oct 1993.
[21] C. Lu, J. Teng, and W.-H. Liu, "Distribution system state estimation," IEEE Trans. on Power Systems, vol. 10, no. 1, pp. 229-240, Feb 1995.

[22] W. Kersting, "Radial distribution test feeders," in Power Engineering Society Winter Meeting, IEEE, vol. 2, 2001, pp. 908-912.

[23] A. Ellis, R. Nelson, E. Von Engeln, R. Walling, J. McDowell, L. Casey, E. Seymor, W. Peter, C. Barker, and B. Kirby, "Reactive power interconnection requirements for PV and wind plants - recommendations to NERC," Sandia Report, Tech. Rep., 2012. 